\title{
Influence of the Matrix on Analyte \\ Fragmentation in Atmospheric Pressure MALDI
}

\author{
E. Schulz and M. Karas \\ Institute of Pharmaceutical Chemistry, Johann-Wolfgang Goethe University Frankfurt, Frankfurt am Main, \\ Germany
}

\author{
F. Rosu and V. Gabelica \\ Mass Spectrometry Laboratory, University of Liège, Liège, Belgium
}

\begin{abstract}
In this paper, we report the measurement of the degree of analyte fragmentation in AP-MALDI as a function of the matrix and of the laser fluence. The analytes include $\mathrm{p}-\mathrm{OCH}_{3}$-benzylpyridinium, three peptides containing the sequence EEPP (which cleave very efficiently at the E-P site), and three deoxynucleosides ( $\mathrm{dA}, \mathrm{dG}$, and $\mathrm{dC}$ ), which lose the neutral sugar to give the protonated base. We found that the matrix hardness/softness was consistent when comparing the analytes, with a consensus ranking from hardest to softest: $\mathrm{CHCA} \gg \mathrm{DHB}>\mathrm{SA} \approx \mathrm{THAP}$ $>\mathrm{ATT}>\mathrm{HPA}$. However, the exact ranking can be fluence-dependent, for example between ATT and HPA. Our goal here was to provide the scientific community with a detailed dataset that can be used to compare with theoretical predictions. We tried to correlate the consensus ranking with different matrix properties: sublimation or decomposition temperature (determined using thermogravimetry), analyte initial velocity, and matrix proton affinity. The best correlation was found with the matrix proton affinity. (J Am Soc Mass Spectrom 2006, 17, 1005-1013) (C) 2006 American Society for Mass Spectrometry
\end{abstract}

$\mathrm{T}$ The most exciting innovations in the last 15 years for the analysis of nonvolatile, high molecular weight compounds like peptides, proteins, oligonucleotides, carbohydrates, and synthetic polymers came from the development of two new ionization techniques for mass spectrometry: matrix-assisted laser desorption/ionization (MALDI) [1, 2] and electrospray ionization (ESI) [3]. MALDI has established itself as a powerful analytical technique. However, despite its wide range of applications and many fundamental studies (for review articles, see [4-7]), there is still a need for better understanding of the MALDI process, to improve ion yields, and to provide rational guidelines for matrix selection. The currently most widely used matrices were found empirically. An important step in the research for new (tailor-made) matrices is an improved knowledge of the role of the matrix in MALDI, and the establishment of criteria to predict matrix properties. The role of the matrix is 3-fold. (1) The matrix has to absorb the laser energy (via electronic excitation in case of UV-MALDI or vibrational excitation in case of IR-MALDI). (2) Disintegration of the condensed phase has to take place without excessive

Published online May 19, 2006

Address reprint requests to Dr. V. Gabelica, Department of Chemistry, Mass Spectrometry Laboratory, University of Liège, Institut de Chimie, Bat. B6c, B-4000 Liège, Belgium. E-mail: v.gabelica@ulg.ac.be destructive heating of the embedded analyte molecules. (3) An efficient ionization of the analyte molecules has to be provided.

It is well known experimentally that MALDI matrices can cause more or less analyte fragmentation, and matrices are therefore characterized as "hard" or "soft", respectively. While ionization with minimal internal energy deposition ("softness") is required for molecular mass determination, extensive fragmentation ("hardness") is advantageous for structural studies, and particularly for peptide sequencing. In addition to application issues, the study of the matrix influence on analyte fragmentation can also bring new elements to the picture of the MALDI mechanism. Several groups have therefore put some effort into the experimental characterization of ion internal energy in MALDI [8-14], or to elaborate MALDI models taking ion internal energy or plume temperature into account [15-20]. Analyte internal energy can be modified by collisional heating and by electronic or vibrational energy-transfer from the matrix, and the study of analyte fragmentation will hopefully bring complementary information on these processes. The main problem in investigating MALDI mechanisms is that MALDI is a complex physical and chemical process, happening in the nanosecond time scale. A dense plume of material containing protonated, deprotonated, and neutral matrix molecules, matrix radicals, electrons [21], hydrogen atoms [22], and the 
analyte is formed and is expanding into the mass spectrometer ion source. The most common way to investigate MALDI mechanisms is via information provided in the mass spectra. While this approach is experimentally convenient, it does not give a direct probe of MALDI. The spectrum is a snapshot of the ion population remaining some microseconds after the laser pulse, but does not provide time resolution on the MALDI mechanism time scale.

In a previous paper [13], we started the investigation of the different contributions to internal energy build-up in MALDI. We have shown that, in vacuumMALDI, activation could occur either due to primary processes (upon laser irradiation) or due to secondary processes (collision with the matrix plume during the pulsed extraction). These two processes are independent; but both are influenced by the choice of the matrix. We also have shown that atmospheric pressure MALDI (AP-MALDI) allows one to study the sole contribution of the primary processes, as the collisions at atmospheric pressure cool down the ions shortly after they have been formed and leave the target, and prevent high-energy CID in the plume. The corresponding fragments can only be formed very early in the expanding plume. Moreover, AP-MALDI gives far more reproducible results than conventional vacuum-MALDI-TOF in terms of fragmentation extent. The study of analyte fragmentation in AP-MALDI can therefore bring new information on the fundamental processes in MALDI and on the role of the matrix in energy-transfer.

In the present paper, we explore the correlation between matrix hardness/softness and different parameters like sublimation/decomposition temperatures, analyte initial velocity, or exothermicity of the gasphase proton affinity. Which matrix property(ies) can be used to rationalize and predict the extent of analyte fragmentation compared to the amount of intact precursor molecules is the key question. We tested six common matrices and used two classes of protonated molecules (deoxynucleosides and peptides) as probe molecules, in addition to the benzylpyridinium cation used before [13].

\section{Methods}

\section{Materials}

Three classes of analytes have been investigated here:

1. Benzylpyridinium ions (quaternary ammonium ions), which allow for the calculation of the ion effective temperature. Compounds were synthesized by the corresponding benzyl chlorides and pyridine (all from Sigma-Aldrich, Dreisenhofen, Germany).

2. Protonated deoxynucleosides (from Sigma-Aldrich).

3. Fragile protonated peptides containing the sequence EEPP. The high fragmentation efficiency of this sequence has been reported by Maux et al. [23]. A 10-amino acid peptide "EP10", with sequence SEEPPAFGKL-[ $\left[\mathrm{NH}_{2}\right]$, was kindly donated by $\mathrm{C}$. Enjalbal (LAPP, University Montpellier II, France) and showed encouraging results. The other 8- and 5-amino acid peptides, "EP8" (SEEPPAKL-[NH $\left.\mathrm{NH}_{2}\right]$ ) and "EP5" (EEPPK-[NH $\left.\mathrm{N}_{2}\right]$ ), were chosen to have a panel of three fragile peptides with different masses. All experiments on the EEPP peptides shown in the present work were performed using peptides purchased from Sigma. All data presented here have been recorded using the peptides from Sigma.

The matrices studied here are: CHCA: $\alpha$-cyano-4hydroxycinnamic acid, from Sigma-Aldrich; DHB: 2,5dihydroxybenzoic acid, from Acros Organics (Geel Belgium), sinapinic acid (3,5-dimethoxy-4-hydroxycinnamic acid), from Sigma-Aldrich (98\%), and Applied Biosystems, (Darmstadt, Germany) ("ultra pure," lot 0205007); THAP: 2', $4^{\prime}, 6^{\prime}$-trihydroxyacetophenone, from Aldrich; ATT: 6-azathiothymine; HPA: 3-hydroxypicolinic acid, from Sigma-Aldrich.

\section{AP-MALDI}

An Agilent ion trap mass spectrometer (1100 series LC/MSD trap XCT, Agilent Technologies, Waldbronn, Germany) equipped with an AP-MALDI source was used for analysis. The nitrogen laser $(337 \mathrm{~nm})$ produces a $10 \mathrm{~Hz}$ pulsed beam, with a pulse width of $\sim 3 \mathrm{~ns}$. The spot size of the laser focus after passing the fibre optics was measurer with thermo sensitive paper. The area of the irradiated surface is $(2.59 \pm 0.08) \times 10^{-3} \mathrm{~cm}^{2}$. The laser energy at used attenuator settings was measured with a NOVA laser power/energy monitor (Ophir Optronics, Rohrsen, Germany) at the level of the target in the opened ion source. With these data, the laser fluence could be calculated for different attenuator settings.

Sample concentration was $10^{-4} \mathrm{~mol} / \mathrm{L}$ for all analyte solutions. Matrices were saturated solutions in water/ acetonitrile 1:1. Matrix and analyte solutions were mixed 1:1 and $1 \mu \mathrm{L}$ of mixture was spotted on the MALDI plate. We checked that our results are independent of matrix-to-analyte ratio in that concentration range. All mass spectra were accumulated during 2 min. The ions and neutrals formed during the MALDI event are dragged through the stainless steal capillary extension toward the quartz-glass capillary. A potential difference of $-4 \mathrm{kV}$ was applied between sample target and inlet capillary. A counter current stream of dry nitrogen was applied and the transfer capillary temperature was set to $150{ }^{\circ} \mathrm{C}$. The capillary exit voltage was set to $50 \mathrm{~V}$, the skimmer to $15 \mathrm{~V}$, and the d.c. voltages on the octapole 1 and 2 to 8.0 and $2.5 \mathrm{~V}$, respectively. The maximum accumulation time during which ions were gated into the trap was set to $600 \mathrm{~ms}$. The actual accumulation time was determined by the ion charge control (ICC), which was set to limit the abundance to 30,000 counts. The trap scanned from 50 to $500 \mathrm{~m} / \mathrm{z}$ in 
the case of benzylpyridinium ions and deoxynucleosides. For the EEPP-peptides, the nitrogen temperature was set to $200{ }^{\circ} \mathrm{C}$, the mass range $300-1300 \mathrm{~m} / \mathrm{z}$, the ICC to 200,000 counts, and the maximum accumulation time was set to $935 \mathrm{~ms}$. The trap drive value (arbitrary units) sets the rf field strength in the ion trap while ions are being accumulated in the trap. The higher the trap drive value, the better the trapping of higher $m / z$ is. The trap drive was set to 25 in the case of benzylpyridinium ions and deoxynucleosides, and 53 in the case of the EEPP peptides to avoid mass-dependent discrimination.

The experimental survival yields were determined from the relative intensities of the parent and fragment ions using eq 1.

$$
S Y_{\exp }-\frac{\mathrm{I}\left(\mathrm{M}^{+}\right)}{\mathrm{I}\left(\mathrm{M}^{+}\right)+\mathrm{I}\left(\mathrm{F}^{+}\right)}
$$

For each combination of matrix/analyte/fluence, the survival yields were independently measured on at least four different spots, and error bars reported below are the standard deviations on these measurements. The points corresponding to the lowest fluence often have a larger error bar because of the low signal-tonoise ratio at ionization threshold.

\section{Thermogravimetry}

Thermogravimetry analyses were performed using a NETZSCH STA 449C. The measurements were done under atmospheric pressure in air. The sample is placed in a platinum crucible. The recorded temperature program started at 20 to $700{ }^{\circ} \mathrm{C}$ with a heating rate of $2{ }^{\circ} \mathrm{C} /$ min unless otherwise mentioned.

\section{Calculation of the Ion Effective Temperature for the Benzylpyridinium Cations}

A complete description of the calculation of the ion effective temperatures from the ion survival yields can be found in the literature [24, 25]. Briefly, the dissociation rate constants $k(E)$ are calculated with the RRKM equation (eq 2).

$$
k(E)=\frac{G^{\ddagger}\left(E-E_{0}\right)}{h N(E)}=\frac{\int_{0}^{E-E_{0}} N^{\ddagger}\left(E_{u}\right) d E_{u}}{h N(E)}
$$

Where $E_{0}$ is the difference between the zero point energy of the transition-state and the zero point energy of the reactant, $G^{\ddagger}\left(E-E_{0}\right)$ is the number of states of the transition-state whose energy lies in the range $[0, E-$ $\left.E_{0}\right], h$ is the Plank constant, and $N(E)$ is the density of states of the reactant at energy $E$. The threshold energies $E_{0}$ for fragmentation were calculated as the energy difference between the zero-point energy of the fragments (pyridine + benzyl) and the zero-point energy of the benzylpyridinium ion. The transition-state was as- sumed to be loose. The density of states $N(E)$ was computed by direct state count. The relationship between the survival yield and the effective temperature is calculated by eq 3 , where $P\left(E ; T_{\text {eff }}\right)$ is the Boltzmann distribution at the temperature $T_{\text {eff }}$ (eq 4 ), and the dissociation time $\tau$ was assumed to be $100 \mathrm{~ns}$.

$$
\begin{aligned}
& S Y\left(T_{\text {eff }}\right)=\int_{0}^{\infty} P\left(E ; T_{\text {eff }}\right) e^{-k(E) \cdot \tau} d E \\
& P(E ; T)=\frac{N(E) e^{-E k T}}{\int_{0}^{\infty} N(E) e^{-E k T} d E}
\end{aligned}
$$

This time-scale of $100 \mathrm{~ns}$ has been chosen quite arbitrarily, as the time required before cooling by collisions at atmospheric pressure is not known [13]. We used the same time-scale as for the vacuum-MALDI study of Luo et al. [26] for purposes of comparison.

\section{Calculation of the Matrix Proton Affinities (PAs)}

Bourcier and Hoppilliard [27] have shown that B3LYP DFT molecular orbital approach allows one to calculate thermochemical properties of MALDI matrices, including DHB and HPA. We used the same approach to calculate the PA of the six matrices investigated. Starting geometries were obtained after a conformational search using Spartan O4 (Wavefunction: www.wavefun.com) software using AM1 level theory. Calculations were carried out using the Gaussian 03 (Gaussian 03, revision B.04, M. J. Frisch et al., Gaussian, Inc. Pittsburgh, PA, 2003) program packages. Geometry optimization and vibrational frequency calculations were carried out at the B3LYP/6-331 $+\mathrm{G}(\mathrm{d}, \mathrm{p})$ level. Final energy was obtained with B3LYP/6-331 ++ G(2d,2p) functional on the optimized geometries. The proton affinity of the matrix $\mathrm{M}$ is defined as PA $(\mathrm{M})=-\Delta \mathrm{H}^{\circ}(\mathrm{M}$ $\left.+\mathrm{H}^{+} \rightarrow \mathrm{MH}^{+}\right)$, and is calculated using eq 5 .

$$
\mathrm{PA}(\mathrm{M})=\Delta \mathrm{E}_{\text {elec }}+\Delta \mathrm{E}_{\text {therm }}(298 \mathrm{~K})+(3 / 2) 298 \mathrm{R}
$$

\section{Results}

\section{Deoxynucleosides}

Deoxynucleosides were chosen because they are quite fragile in their protonated form. After the cleavage of the glycosidic bond the protonated base appears in the spectra as the fragment signal. 2'-deoxyadenosine molecular ion $[\mathrm{M}+\mathrm{H}]^{+}$is observed at $252 \mathrm{~m} / \mathrm{z}$ and its fragment ion at $136 \mathrm{~m} / \mathrm{z}, 2^{\prime}$-deoxycytosine molecular ion $[\mathrm{M}+\mathrm{H}]^{+}$is observed at $228 \mathrm{~m} / \mathrm{z}$ and its fragment ion at $112 \mathrm{~m} / \mathrm{z}, 2^{\prime}$-deoxyguanosine molecular ion $[\mathrm{M}+\mathrm{H}]^{+}$is observed at $268 \mathrm{~m} / \mathrm{z}$ and its fragment ion at $152 \mathrm{~m} / \mathrm{z}$. Experimentally measured survival yields (eq 1) as a function of the laser fluence and for the different matrices are shown in Figure 1. 

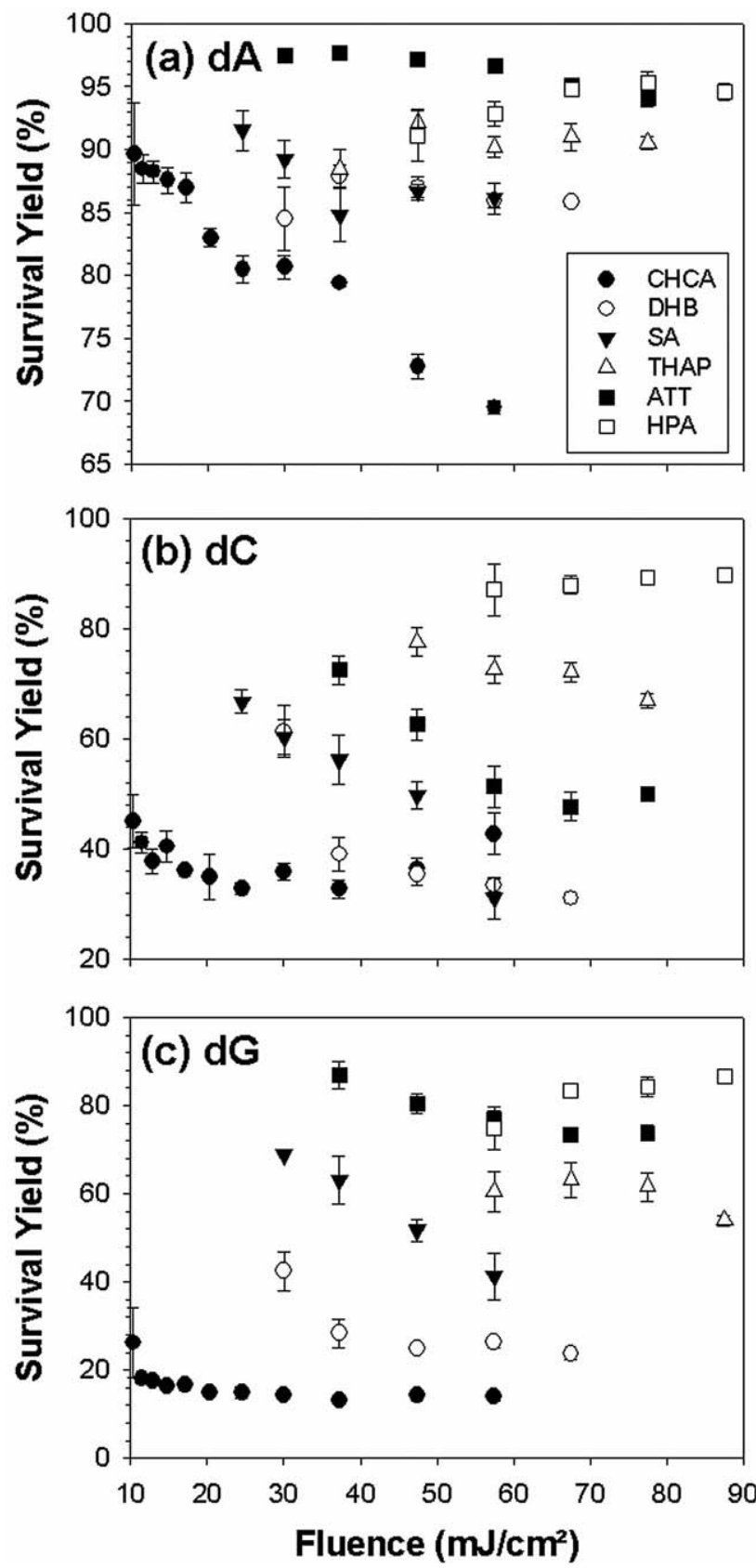

Figure 1. Experimentally measured survival yields (eq 1) of (a) 2'-deoxyadenosine (dA), (b) 2'-deoxycytosine (dC), and (c) 2'deoxyguanosine $(\mathrm{dG})$ as a function of the laser fluence for the different matrices. The symbol legend in the inset of panel (a) holds for all graphs.

The data do not exhibit the exact same behavior for the three deoxynucleosides. For 2'-deoxycytosine, we encountered two experimental problems that influence the determined survival yields: (1) 2'-dC peaks have interferences with matrix peaks in the cases of ATT (interference with fragment at $122 \mathrm{Da}$ ) and SA (interference with precursor at $228 \mathrm{Da}$ ), and (2) $2^{\prime}$-dC undergoes reductive hydrogenation with $\mathrm{DHB}$ (see Figure 2). This behavior has already been reported [28]. Therefore, for 2 '-dC (Figure $1 b)$, only a partial ranking can be estab- lished $(\mathrm{CHCA}>\mathrm{ATT}>\mathrm{THAP}>\mathrm{HPA})$. In the case of deoxynucleosides, fragmentation increases with the laser fluence, except for HPA.

There are several ways to establish a matrix ranking based on their hardness/softness. One could consider matrix hardness at their ionization threshold, the mean hardness over the entire fluence range, the hardness at the fluence that gives best signal-to-noise, or the matrix behavior at the fluence corresponding to the softest conditions. The choice of the fluence depends on the needs of the experimentalist for each particular application. Here, the complete set of datapoints is provided so the reader can examine the results on his own point of view. Overall, for the deoxynucleosides the ranking of matrices, from hardest to softest, is $\mathrm{CHCA}>\mathrm{DHB}>$ $\mathrm{SA}>\mathrm{THAP}>\mathrm{ATT} \geq \mathrm{HPA}$.

\section{Peptides Containing the Sequence EEPP}

To test the effect of the matrix on the extent of fragmentation of protonated peptides (the analytes of interest for proteomics applications), a particularly fragile peptide with preferably one cleavage site was needed. Most peptides do not yield sufficiently intense fragmentation under the soft conditions of AP-MALDI. Maux et al. [23] reported that peptides containing two consecutive proline residues in their sequence exhibit a specific abundant backbone cleavage N-terminal to proline, and that peptides containing the sequence EEPP were particularly fragile. We used three peptides containing the sequence "EEPP" as probes for fragmentation efficiency. The APMALDI spectrum of peptide EP10 in the hardest matrix (CHCA) is shown in Figure 3. The cleavage between $\mathrm{E}$ and $\mathrm{P}$ can be observed for all the matrices, and it is the major fragmentation pathway observed. The survival yields were measured as a function of the laser fluence for all six matrices for the peptides EP10, EP8, and EP5, considering the major $y$ ion as the fragment, and the results are shown in Figure 4. For EP10 (which gives the most efficient fragmentation), matrix ranking from hardest to softest is: $\mathrm{CHCA}>\mathrm{DHB}>\mathrm{SA}>\mathrm{THAP}>\mathrm{ATT}>\mathrm{HPA}$. For the peptides EP8 and EP5, the fragmentation efficiency is lower. The matrix ranking is the same as for EP10, except that 2,5-DHB is now the second softest matrix after HPA, and is the softest if one considers matrix behavior at ionization threshold. A particular and yet unexplained phenomenon is observed for CHCA: survival yields first decrease rapidly with laser fluence, but then increase again at fluences larger than $20 \mathrm{~mJ} / \mathrm{cm}^{2}$. One should not forget that both the effective temperature and the fragmentation time play a role in the survival yield (see eq 3 ), but in the present experiments we have no evidence on which parameter is at the origin of this behavior.

\section{Benzylpyridinium Ions}

These cations have been extensively used by different groups to characterize the ion internal energy in various ionization methods (for a review, see [24]). The advan- 


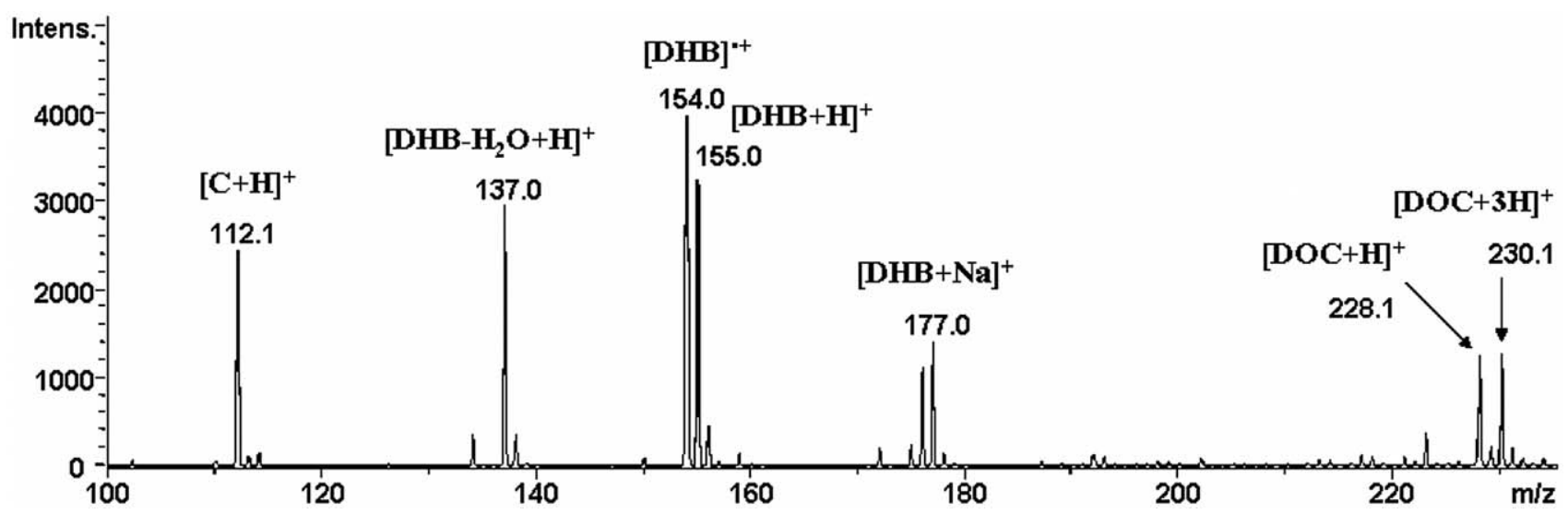

Figure 2. Mass spectrum of $2^{\prime}-\mathrm{dC}$ (noted DOC for deoxycytosine) in the matrix DHB with a laser fluence of $67.5 \mathrm{~mJ} / \mathrm{cm}^{2}$. Parent ion peak $[\mathrm{M}+\mathrm{H}]^{+}$is at $228 \mathrm{Da}$, hydrogenated form $[\mathrm{M}+3 \mathrm{H}]^{+}$is observed at $230 \mathrm{Da}$, and fragment (protonated cytidine, noted $[\mathrm{C}+\mathrm{H}]^{+}$) at $112 \mathrm{Da}$.

tage of this "thermometer molecule" is that an effective temperature can be calculated from the survival yield. For a 100 ns fragmentation time, the survival yield calculated at each effective temperature is shown in Figure 5 for three benzylpyridiniums used in our previous work in AP-MALDI with CHCA, DHB, and SA [13]. These curves have been used in the present work for all conversions from the measured survival yield to the corresponding effective temperature.

In our previous work, the matrix effective temperatures (SA, DHB, and CHCA) were lying between 800 and $1100 \mathrm{~K}$. Here we included matrices that are reputed to be even softer (ATT and HPA). It can be clearly seen on Figure 5 that only the benzylpyridinium with the $\mathrm{p}-\mathrm{OCH}_{3}$ substituent can give significant fragmentation in the cases of soft matrices (low effective temperature). In effect, we could not detect measurable fragment ion signal in the case of $\mathrm{p}-\mathrm{CH}_{3}$ and $\mathrm{p}-\mathrm{tBu}$ and the softest matrices. Therefore, for the present work, we used only the probe molecule $\mathrm{p}-\mathrm{OCH}_{3}$ to compare the six matrices under study. The results are presented in Figure 6. Considering the mean effective temperatures on the whole fluence range, the ranking of matrices, from hardest to softest, is: CHCA $(1031 \mathrm{~K})>$ DHB $(921 \mathrm{~K})>$ SA $(888 \mathrm{~K})>$ THAP $(872 \mathrm{~K})>\operatorname{ATT}(825 \mathrm{~K})>\mathrm{HPA}(810$ $\mathrm{K})$. At matrix ionization threshold, however, the ordering is slightly changed: CHCA $(988 \mathrm{~K})>\mathrm{DHB}(921 \mathrm{~K})$ $>$ THAP $(904 \mathrm{~K})>$ SA $(887 \mathrm{~K})>\mathrm{HPA}(808 \mathrm{~K})>\mathrm{ATT}$ $(800 \mathrm{~K})$. Again, this illustrates that apparent matrix hardness depends on the experiment purpose and design.

\section{Thermogravimetry}

A correlation between matrix hardness and sublimation temperature would indicate that the extent of analyte fragmentation depends on the peak temperature reached in the matrix before explosion and desorption. We therefore have performed thermogravimetric measurements at atmospheric pressure and under air. An endothermic peak accompanied by a significant mass loss is indicative of sublimation. When the mass loss is not $100 \%$, we interpret the reaction as decomposition with released gas rather than sublimation. Thermogravimetric results at atmospheric pressure are collected in Table 1.

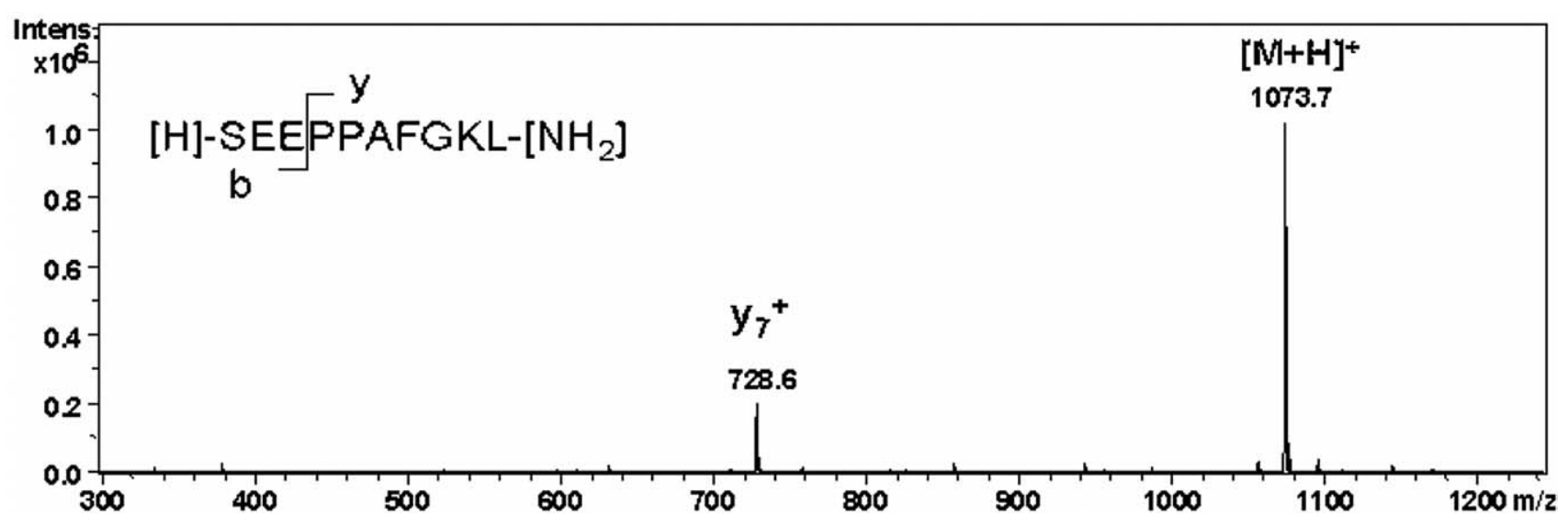

Figure 3. Sequence of the peptide EP10 and mass spectrum of the peptide EP10 in CHCA with the laser fluence of $22 \mathrm{~mJ} / \mathrm{cm}^{2}$. 
(a)

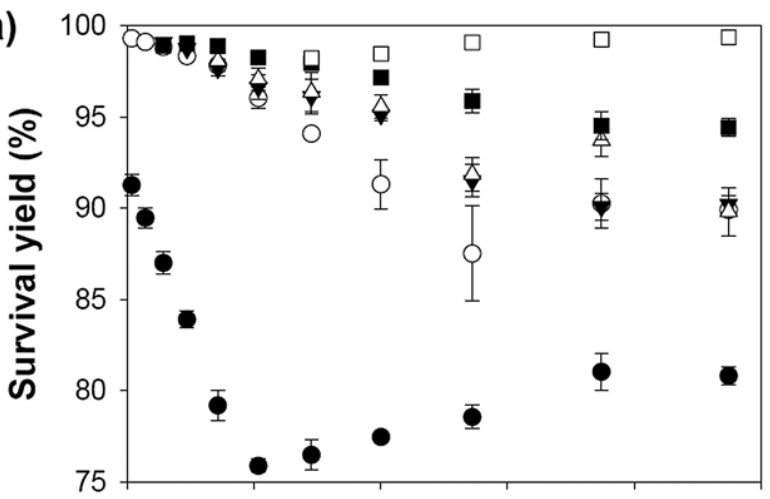

(b)
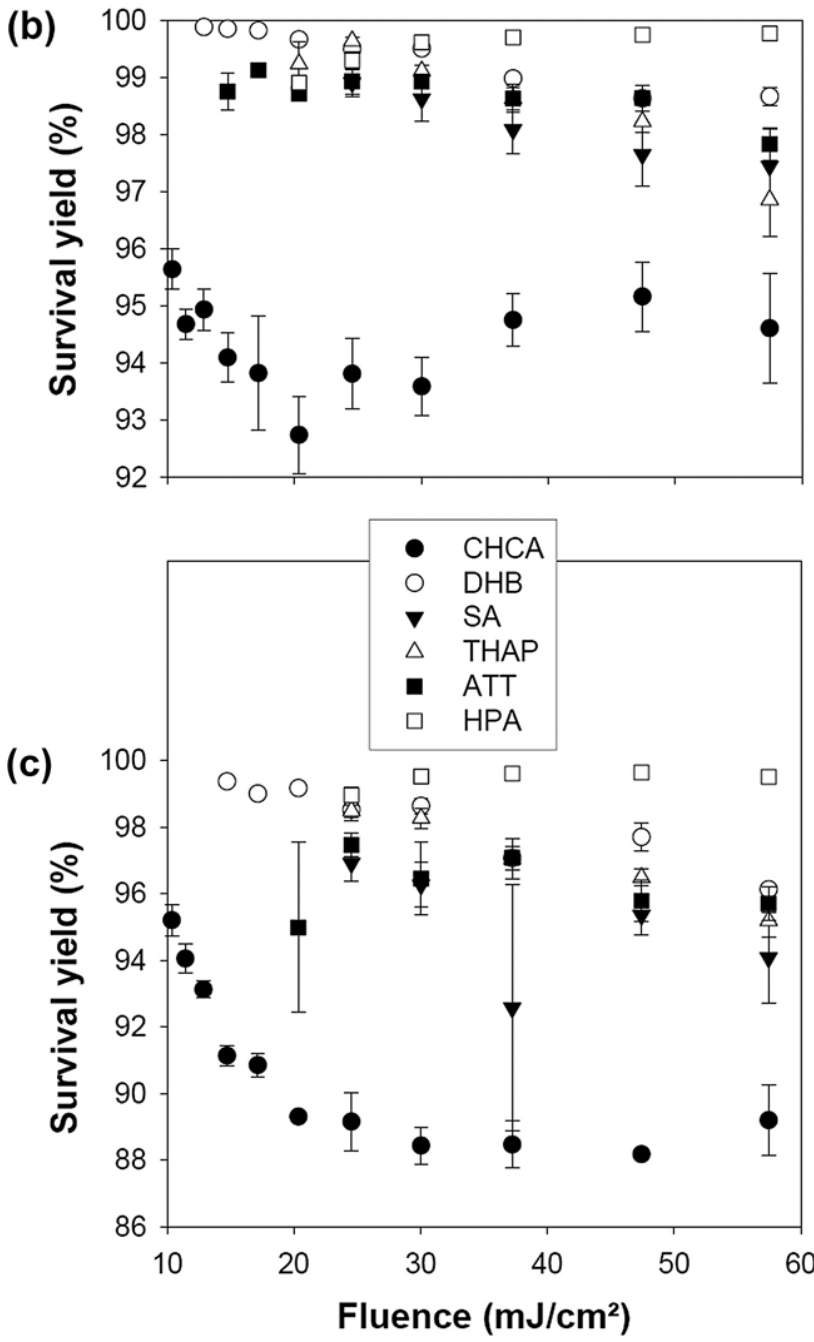

Figure 4. Experimentally measured survival yields (eq 1) for the peptides (a) EP10, (b) EP8, and (c) EP5 as a function of the laser fluence for the different matrices. The symbol legend in the inset of panel (c) holds for all graphs. Large error bars are usually due to the low signal-to-noise ratios of the parent and fragment ions.

There is apparently no clear correlation between the transition temperatures and the matrix hardness/softness. HPA, which is the softest matrix, followed by ATT, have higher transition temperatures than SA. However, we found in the case of some matrices a dependence of the transition temperature on the heat-

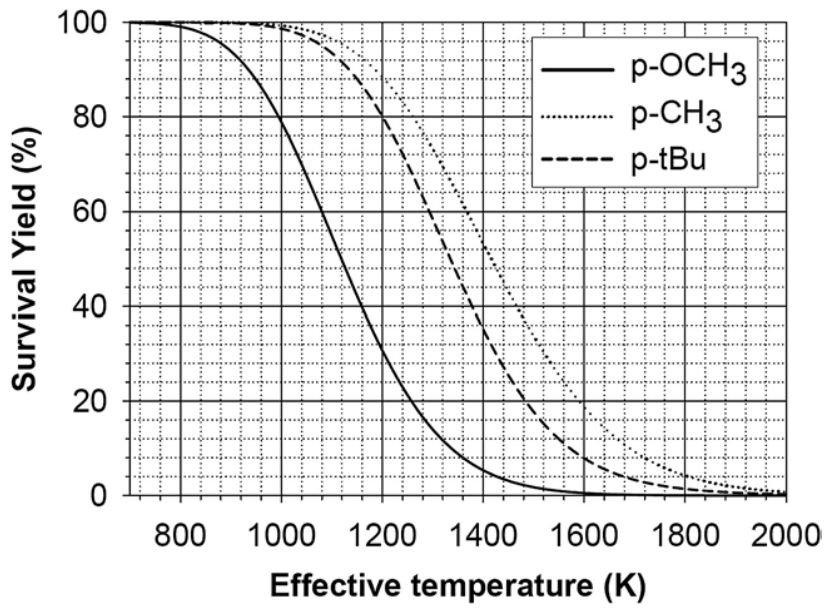

Figure 5. Survival yields of the benzylpyridinium cations, calculated as a function of the effective temperature, for a reaction time $\tau$ of 100 ns.

ing rate. At $5{ }^{\circ} \mathrm{C} / \mathrm{min}$, the transition temperatures increased, for example in the case of CHCA and ATT. Compared to the speed of the MALDI process, these heating rates are still very low, and in MALDI a

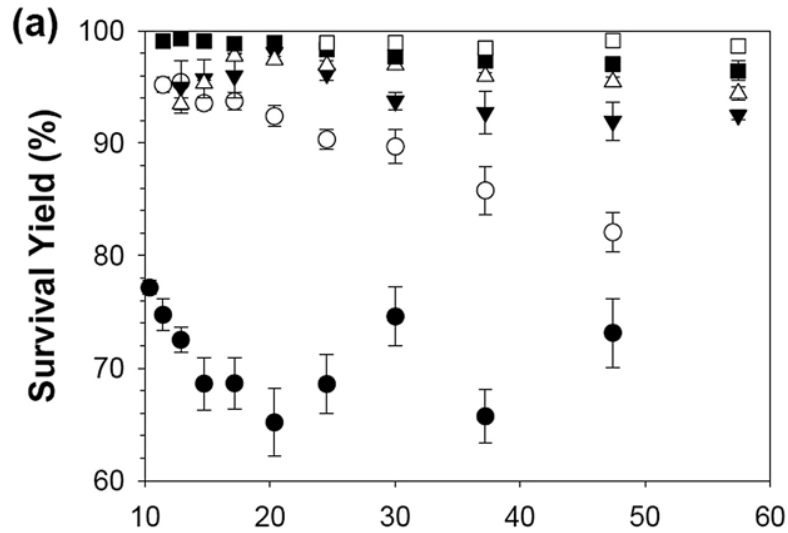

(b) Fluence $\left(\mathrm{mJ} / \mathrm{cm}^{2}\right)$

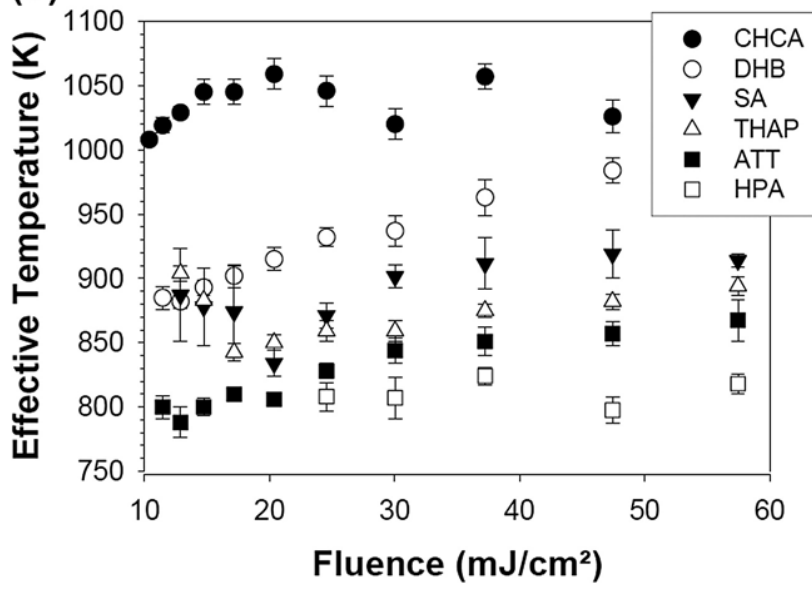

Figure 6. (a) Survival yields and (b) effective temperatures of p-OCH $\mathrm{OCH}_{3}$ as a function of the laser fluence for the six different matrices. The symbol legend in the inset of panel $(\mathbf{b})$ holds for both graphs. 
Table 1. Thermogravimetry results

\begin{tabular}{lrrrr}
\hline Matrix & $\Delta$ Mass 1 & Transition temperature 1 (sharp) & $\Delta$ Mass 2 & Temperature range 2 (broad) \\
\hline \hline CHCA & $-70 \%$ & $251^{\circ} \mathrm{C}$ & $-30 \%$ & $370 \rightarrow 520^{\circ} \mathrm{C}$ \\
DHB & $-100 \%$ & $207^{\circ} \mathrm{C}$ & $-40 \%$ & $200 \rightarrow 430^{\circ} \mathrm{C}$ \\
SA & $-60 \%$ & $187^{\circ} \mathrm{C}$ & $-50 \%$ & $240 \rightarrow 500^{\circ} \mathrm{C}$ \\
THAP & $-50 \%$ & $222^{\circ} \mathrm{C}$ & & \\
ATT & $-100 \%$ & $222^{\circ} \mathrm{C}$ & & \\
HPA & $-100 \%$ & $198^{\circ} \mathrm{C}$ & & \\
\hline
\end{tabular}

significant degree of metastable heating is to be expected. More detailed experimental insight into the temperatures attained in fast heating rather than slow equilibrium heating would be extremely insightful in the MALDI case.

\section{Calculation of Matrix PA}

Another correlation that has been suggested previously $[6,9]$ for the analyte internal energy is a correlation with the exothermicity of the matrix-to-analyte proton transfer reaction. In positive ion mode, the exothermicity is correlated with the matrix proton affinity (PA). For the reaction

$$
\text { Analyte }+ \text { MatrixH }^{+} \rightarrow \text { AnalyteH }^{+}+\text {Matrix }
$$

$\Delta \mathrm{H}^{\circ}=\mathrm{PA}$ (matrix) - PA (analyte). Therefore low PA matrices would give more internal energy $\left(\Delta \mathrm{H}^{\circ}<0\right)$.

Matrix PA can be determined experimentally by the kinetic method $[29,30]$ or by B3LYP DFT calculations in the case of DHB and HPA [27, 31]. We performed such calculations for the four other matrices under study (see eq 5 in the Methods section). For DHB and HPA, the calculated values are in good agreement with the experimental values. However, larger discrepancies are found for the larger matrices like CHCA and SA, suggesting that even the high level calculations performed is not sufficient to compute accurately the proton affinities. Table 2 summarizes the proton affinity values from different sources. Interestingly, the matrix hardness is inversely correlated with the matrix proton affinity (as determined by Mirza et al. [30] using the kinetic method).

\section{Discussion}

Among all three classes of substances (all positive ions) there is a relatively good agreement regarding

Table 2. Matrix proton affinities (PA), determined either by calculation or experimentally by the kinetic method

\begin{tabular}{lccc}
\hline Matrix & $\begin{array}{c}\text { PA (kJ/mol) } \\
\text { Calculations }\end{array}$ & PA (kJ/mol) [29] & PA (kJ/mol) [30] \\
\hline \hline CHCA & 812 & 841.5 & 841.5 \\
DHB & $852[27]$ & - & 856 \\
SA & 905 & 887.6 & 876 \\
THAP & 899 (on C $=0)$ & - & 893 \\
ATT & 842 (on S) & - & - \\
HPA & $903[27]$ & 896 & 898.5 \\
\hline
\end{tabular}

matrix hardness/softness. The consensus ranking from hardest to softest is: $\mathrm{CHCA} \gg \mathrm{DHB}>\mathrm{SA} \approx$ THAP $>$ ATT $>$ HPA. But there are also some more subtle variations according to the laser fluence (e.g., for HPA, there is more fragmentation at ionization threshold than at high fluences, so that ATT is softer than HPA at ionization threshold) and to the analyte (e.g., in the case of the EP8 and EP5 peptides, DHB is found the second softest behind HPA). This is probably the reason why there was, till now, no clear consensus in the literature on the matrix rankings. Nevertheless, the understanding of the origins of matrix hardness/softness is believed to bring more insight into the MALDI process, i.e., how the laser energy is transferred and partitioned into translational energy (desorption) and internal energy (causing fragmentation).

If one imagines a simple model where the analyte internal energy depends on the temperature reached by the matrix before expansion starts, then the analyte internal energy should be proportional to the sublimation temperature (or to the decomposition temperature if the gas release is at the origin of matrix explosion). The correlation works only very roughly: CHCA, the hardest matrix, has indeed the highest sublimation temperature, but the ranking of the other matrices does not follow the trend expected from this model. However, as discussed above, the local laser-induced matrix heating is very sudden and, therefore, the correlation with equilibrium data (the temperature of phase transition) is not the most adequate. The internal temperature reached by analytes and clusters upon MALDI has been addressed by computer simulations [7, 32], but one still lacks experimental data on local temperatures reached with fast heating, giving rise to a metastable hot spot just before expansion and desorption.

Another matrix parameter that has previously been proposed to be correlated to analyte internal energy is the initial velocity of the analytes desorbed with that matrix [33, 34]. The proposed model was that the internal energy depends on the balance between translational energy and internal energy. If the plume expands at higher velocity, this is at the expense of the internal energy. It has been shown that the analyte initial velocity is matrix-dependent, but not analytedependent $[33,34]$. It is interesting to note that in our experiments, matrix hardness is also not very analytedependent. Analyte initial velocities reported for the six matrices studied are: $311 \mathrm{~m} / \mathrm{s}$ [35] or $291 \mathrm{~m} / \mathrm{s}$ [34] for 
CHCA, $543 \mathrm{~m} / \mathrm{s}$ [34] for DHB, $352 \mathrm{~m} / \mathrm{s}$ [35] or $332 \mathrm{~m} / \mathrm{s}$ [34] for SA, $306 \mathrm{~m} / \mathrm{s}$ [34] for THAP, $315 \mathrm{~m} / \mathrm{s}$ [34] for ATT, $537 \mathrm{~m} / \mathrm{s}$ [35], 444 or $620 \mathrm{~m} / \mathrm{s}$ [34] for HPA (444 in water and 620 in water/ethanol).

Again, the correlation works well in some cases (e.g., CHCA versus $\mathrm{DHB}$ ), but is not perfect. The large difference in matrix hardness between CHCA and the others cannot be accounted for by the differences in initial velocity. HPA in some experimental conditions has given rise to very high initial velocities, and HPA is also the softest matrix. ATT, on the contrary, has a low initial velocity, but is softer than DHB except for two of the three peptides studied here. For these two peptides (EP5 and EP8), the correlation between matrix hardness and the initial velocity as determined by Glückmann et al. [34] is good.

The matrix property that best correlates with the fragmentation extent of the analyte is the matrix proton affinity. This is in agreement with the two-step MALDI model proposed by Knochenmuss, Zenobi and coworkers $[6,36]$ : the first step is the laser absorption by the matrix resulting in a local explosion, expansion, and in the formation of the plume; the second step consists of all the possible reactions occurring in the expanding plume. The final population is the result of these two steps. At fluences higher than threshold, the ion population is under thermodynamic control $[6,36]$. Note that under AP-MALDI the contribution of analyte-matrix collisions is negligible compared to analyte-gas collisions as soon as the dense plume has dissipated. Our results suggest that the most important contribution to analyte final internal energy is due to secondary processes (the proton transfer reactions), as suggested previously from observations in the negative ion mode [9]. This does not mean that other contributions do not exist. Still, a point that remains puzzling is that this excellent correlation between matrix hardness and proton affinity is observed even in the case of the benzylpyridinium cation, which does not result from a direct protonation reaction.

In conclusion, we hope that the experiments reported here on the extent of analyte fragmentation for different analytes as a function of the matrix and of the laser fluence in AP-MALDI will constitute a useful set of experimental data to test the different models proposed. But as for now, we could not infer a "one-fits-all" model for explaining and predicting analyte fragmentation in MALDI. Additional experiments with other classes of permanent cations (quaternary ammonium or phosphonium), or cationized species, could help to solve the contradiction.

\section{Acknowledgments}

Dr. F. Mandel (Agilent Technologies, Waldbronn, Germany) is gratefully acknowledged for providing technical support with AP-MALDI. The authors thank Mrs. Isabelle Lecomte (LCIS, University of Liège, Belgium) for the thermogravimetry analyses, and Dr. C. Enjalbal (LAPP, University Montpellier II, France) for providing a sample of EP10 peptide. VG is a Research Associate of the FNRS (Belgium).

\section{References}

1. Karas M.; Bachmann D.; Bahr U.; Hillenkamp, F. Matrix-assisted ultraviolet laser desorption of nonvolatile compounds. Int. J. Mass Spectrom. Ion Processes 1987, 78, 53-68.

2. Karas M.; Hillenkamp, F. Laser desorption ionization of proteins with molecular masses exceeding 10,000 Daltons. Anal. Chem. 1988, 60, 2299-2301

3. Yamashita M.; Fenn, J. B. Electrospray ion source: Another variation on the free-jet theme. J. Phys. Chem. 1984, 88, 4451-4459.

4. Dreisewerd, K. The desorption process in MALDI. Chem. Rev. 2003, 103, 395-425.

5. Karas M.; Krüger, R. Ion formation in MALDI: The cluster ionization mechanism. Chem. Rev. 2003, 103, 427-439.

6. Knochenmuss R.; Zenobi R. MALDI ionization: The role of in-plume processes. Chem. Rev. 2003, 103, 441-452

7. Zhigilei L. V.; Leveugle E.; Garrison B. J.; Yingling Y. G.; Zeifman, M. I. Computer simulations of laser ablation of molecular solids. Chem. Rev. 2003, 103, 321-347.

8. Mowry C. D.; Johnston, M. V. Internal energy of neutral molecules ejected by matrix-assisted laser desorption. J. Phys. Chem. 1994, 98, 1904-1909.

9. Stevenson E.; Breuker K.; Zenobi, R. Internal energies of analyte ions generated from different MALDI matrices. J. Mass Spectrom. 2000, 35 , 1035-1041.

10. Luo G. H.; Marginean I.; Vertes, A. Internal energy of ions generated by matrix-assisted laser desorption/ionization. Anal. Chem. 2002, 74, 61856190.

11. Greisch J.-F.; Gabelica V.; Remacle F.; De Pauw, E. Thermometer ions for matrix-enhanced laser desorption/ionization internal energy calibration. Rapid Commun. Mass Spectrom. 2003, 17, 1847-1854.

12. Vertes A.; Luo G. H.; Ye L.; Chen Y.; Marginean, I. Laser pulse length dependence of internal energy transfer in UV-MALDI-MS. Materials Science and Processing. Appl. Phys. A 2004, 79, 823-825.

13. Gabelica V.; Schulz E.; Karas, M. Internal energy build-up in matrixassisted laser desorption/ionization. J. Mass Spectrom. 2004, 39, 579-593.

14. Konn D. O. Murrell J · Despeyroux D. Gaskell, S. J. Comparison of the effects of ionization mechanism, analyte concentration, and ion "cooltimes" on the internal energies of peptide ions produced by electrospray and atmospheric pressure matrix-assisted laser desorption ionization. J. Am. Soc. Mass Spectrom. 2005, 16, 743-751.

15. Vertes A.; Irinyi G.; Gijbels, R. Hydrodynamic model of matrix-assisted laser desorption mass spectrometry. Anal. Chem. 1993, 65, 2389-2393.

16. Bencsura A.; Navale V.; Sadeghi M.; Vertes, A. Matrix-guest energy transfer in MALDI. Rapid Commun. Mass Spectrom. 1997, 11, 679-682.

17. Knochenmuss, R. A quantitative model of ultraviolet matrix-assisted laser desorption/ionization. J. Mass Spectrom. 2002, 37, 867-877.

18. Zhigilei L. V.; Leveugle E.; Garrison B. J.; Yingling Y. G.; Zeifman, M. I. Computer simulations of laser ablation of molecular substrates. Chem. Rev. 2003, 103, 321-347.

19. Knochenmuss, R. A quantitative model of ultraviolet matrix-assisted laser desorption/ionization including analyte ion generation. Anal. Chem. 2003, 75, 2199-2207.

20. Knochenmuss R.; Zhigilei, L. V. Molecular dynamics model of ultraviolet matrix-assisted laser desorption/ionization including ionization processes. J. Phys. Chem. B 2005, 109, 22947-22957.

21. Frankevitch V. E.; Zhang J.; Friess S. D.; Dashtiev M.; Zenobi, R. Role of electrons in laser desorption/ionization mass spectrometry. Anal. Chem. 2003, 75, 6063-6067.

22. Calba P. J.; Muller J. F.; Inouye, M. H-atom transfer following analyte photoionization in MALDI process. Rapid Commun. Mass Spectrom. 1998, 12, 1727-1731.

23. Maux D.; Enjalbal C.; Martinez J.; Aubagnac, J.-L. New example of proline-induced fragmentation in electrospray ionization mass spectrometry of peptides. Rapid Commun. Mass Spectrom. 2002, 16, 14701475 .

24. Gabelica V.; De Pauw, E. Internal energy and fragmentation of ions produced in electrospray sources. Mass Spectrom. Rev. 2005, 24, 566-587.

25. Drahos L.; Heeren R. M. A.; Collette C.; De Pauw E.; Vékey, K. Thermal energy distributions observed in electrospray ionization. J. Mass Spectrom.1999, 34, 1373-1379.

26. Luo G.; Marginean I.; Vertes, A. Internal energy of ions generated by matrix-assisted laser desorption/ionization. Anal. Chem. 2002, 74, 61856190.

27. Bourcier S.; Hoppilliard, Y. B. 3LYP DFT molecular orbital approach, an efficient method to evaluate the thermochemical properties of MALDI matrices. Int. I. Mass Spectrom. 2002, 217, 231-244.

28. Koomen J. M.; Russell D. H. UV MALDI MS characterization of 2,5-dihydroxybenzoic acid-indiced reductive hydrogenation of oligonucleotides on cysteine residues. I. Mass Spectrom. 2000, 35, 1025-1034.

29. Jorgensen T. J. D.; Bojesen G.; Rahbeck-Nielsen, H.The proton affinities of seven matrix-assisted laser desorption/ionization matrices correlated with the formation of multiply charged ions.Eur. J. Mass Spectrom.439199845 
30. Mirza S. P.; Raju N. P.; Vairamani, M. Estimation of the proton affinity values of fifteen matrix-assisted laser desorption/ionization matrices under electrospray ionization conditions using the kinetic method. J. Am. Soc. Mass Spectrom. 2004, 15, 431-435.

31. Yassin F. H.; Marynick, D. S. Computational estimates of the gas-phase acidities of dihydroxybenzoic acid radical cations and their corresponding neutral species. J. Mol. Struct. 2004, 629, 223-235.

32. Zhigilei, L. V. Dynamics of the plume formation and parameters of the ejected clusters in short-pulse laser ablation. Appl. Phys. A 2003, 76, $339-350$.

33. Karas M.; Bahr U.; Fournier I.; Glückmann M.; Pfenninger, A. The initial-ion velocity as a marker for different desorption-ionization mechanisms in MALDI. Int. J. Mass Spectrom. 2003, 226, 239-248.
34. Glückmann M.; Karas, M. The initial velocity and its dependence on matrix, analyte, and preparation method in ultraviolet matrix-assisted laser desorption/ionization. J. Mass Spectrom. 1999, 34, 467477.

35. Juhasz P.; Vestal M. L.; Martin, S. A. On the initial velocity of ions generated by MALDI and its effects on the calibration of delayed extraction TOF mass spectra. J. Am. Soc. Mass Spectrom. 1997, 8, 209-217.

36. Breuker K.; Knochenmuss R.; Zhang J.; Stortelder A.; Zenobi, R. Thermodynamic control of final ion distributions in MALDI: Inplume proton transfer reactions. Int. J. Mass Spectrom. 2003, 226, 211-222. 